Macromolecular Nanotechnology

\title{
Glass transition and polymer dynamics in silver/poly(methyl methacrylate) nanocomposites
}

\author{
Ch. Pandis ${ }^{\mathrm{a}, *}$, E. Logakis ${ }^{\mathrm{a}}$, A. Kyritsis ${ }^{\mathrm{a}}$, P. Pissis ${ }^{\mathrm{a}}$, V.V. Vodnik ${ }^{\mathrm{b}}$, E. Džunuzović ${ }^{\mathrm{c}}$, J.M. Nedeljković $^{\mathrm{b}}$, \\ V. Djoković ${ }^{\mathrm{b}}$, J.C. Rodríguez Hernández ${ }^{\mathrm{d}}$, J.L. Gómez Ribelles ${ }^{\mathrm{d}, \mathrm{e}, \mathrm{f}}$ \\ ${ }^{a}$ National Technical University of Athens, Zografou Campus, 15780 Athens, Greece \\ ${ }^{\mathrm{b}}$ Vinča Institute of Nuclear Sciences, University of Belgrade, P.O. Box 522, 11001 Belgrade, Serbia \\ ${ }^{\mathrm{c}}$ Faculty of Technology and Metallurgy, University of Belgrade, Karnegijeva 4, 11120 Belgrade, Serbia \\ ${ }^{\mathrm{d}}$ Centro de Biomateriales e Ingeniería Tisular, Universidad Politecnica de Valencia, P.O. Box 22012, E-46071 Valencia, Spain \\ ${ }^{\mathrm{e}}$ Regenerative Medicine Unit, Centro de Investigación Príncipe Felipe, Autopista del Saler 16, 46013 Valencia, Spain \\ ${ }^{\mathrm{f}}$ Networking Research Center on Bioengineering, Biomaterials and Nanomedicine (CIBER-BBN), Valencia, Spain
}

\section{A R T I C L E I N F O}

\section{Article history:}

Received 8 December 2010

Received in revised form 27 May 2011

Accepted 5 June 2011

Available online 6 July 2011

\section{Keywords:}

Ag nanoparticles

Polymer dynamics

Glass transition

Segmental relaxation

Free volume

\begin{abstract}
A B S T R A C T
Dynamic mechanical-thermal analysis (DMTA), differential scanning calorimetry (DSC), thermally stimulated depolarization currents (TSDC) and, mainly, broadband dielectric relaxation spectroscopy (DRS) were employed to investigate in detail glass transition and polymer dynamics in silver/poly(methyl methacrylate) (Ag/PMMA) nanocomposites. The nanocomposites were prepared by radical polymerization of MMA in the presence of surface modified Ag nanoparticles with a mean diameter of $5.6 \mathrm{~nm}$ dispersed in chloroform. The fraction of $\mathrm{Ag}$ nanoparticles in the final materials was varied between 0 and $0.5 \mathrm{wt} \%$, the latter corresponding to $0.055 \mathrm{vol} \%$. The results show that the nanoparticles have practically no effect on the time scale of the secondary $\beta$ and $\gamma$ relaxations, whereas the magnitude of both increases slightly but systematically with increasing filler content. The segmental $\alpha$ relaxation, associated with the glass transition, becomes systematically faster and stronger in the nanocomposites. The glass transition temperature $T_{\mathrm{g}}$ decreases with increasing filler content of the nanocomposites up to about $10^{\circ} \mathrm{C}$, in good correlation by the four techniques employed. Finally, the elastic modulus decreases slightly but systematically in the nanocomposites, both in the glassy and in the rubbery state. The results are explained in terms of plasticization of the PMMA matrix, due to constraints imposed to packing of the chains by the Ag nanoparticles, and, at the same time, of the absence of strong polymer-filler interactions, due to the surface modification of the Ag nanoparticles by oleylamine at the stage of preparation.
\end{abstract}

(c) 2011 Elsevier Ltd. All rights reserved.

\section{Introduction}

In a recent paper some of us reported on the preparation of silver/poly(methyl methacrylate) (Ag/PMMA) nanocomposites in two steps [1]. First, Ag nanoparticles were synthesized in water and transferred into chloroform using oleylamine as transfer agent. In the second step, the surface modified $\mathrm{Ag}$ nanoparticles were encapsulated in

\footnotetext{
* Corresponding author.

E-mail address: pandis@mail.ntua.gr (Ch. Pandis).
}

PMMA by in situ radical polymerization of methyl methacrylate. Transmission electron microscopy (TEM) measurements on the Ag colloids in chloroform showed that the Ag nanoparticles are spherical with a mean diameter of $5.6 \mathrm{~nm}$. The obtained transparent nanocomposites were characterized using gel permeation chromatography, UVvis spectroscopy, proton NMR spectroscopy, differential scanning calorimetry (DSC), and thermo-gravimetric analysis (TGA). Analysis of the optical measurements indicated the formation of loose Ag aggregates (pairs and squares) in the nanocomposites [1]. 
In the present paper, we employ dynamic mechanical-thermal analysis (DMTA), DSC and two dielectric techniques, thermally stimulated depolarization currents (TSDC) and broadband dielectric relaxation spectroscopy (DRS), to investigate in detail glass transition and polymer dynamics in the Ag/PMMA nanocomposites of Ref. [1]. The broad frequency and temperature ranges of dielectric measurements allow to study on the same samples, in addition to segmental dynamics associated with the glass transition (dynamic glass transition [2]), also secondary (local) relaxations, as well as electrical conductivity.

Next to fundamental interest in dynamics studies in polymer nanocomposites, e.g. in relation to the behavior of a polymer or a liquid in the vicinity of a solid surface [3], such studies are also of practical interest. They form an essential part in the investigation of structure-property relationships. A true understanding of the latter is a prerequisite for optimizing composition and processing conditions for preparing materials with predicted properties tailored to specific applications $[4,5]$.

We may expect that, similar to structure/morphology and various properties, chain dynamics in polymer nanocomposites is modified, as compared to the neat polymer. In fact, computer simulations and experiments with model systems provide evidence that polymeric chains in the vicinity of a solid surface, within a distance of a few nm, exhibit different organization (density, chain configuration) and properties (thermal transitions, molecular mobility), as compared to chains in the bulk $[3,6,7]$. On the other hand, results in real polymer nanocomposites, in particular on segmental dynamics associated with the glass transition, often appear controversial and confusing, as dynamics (often quantified in terms of glass transition temperature $T_{\mathrm{g}}$ ) may become faster or slower or show no change, it may be homogeneous or heterogeneous [8]. Obviously, several factors affect polymer dynamics in the nanocomposites and should be critically taken into account. It has been pointed out that two main contradictory effects of nanoparticles on chain dynamics should be properly considered [9,10]: dynamics may become slower, in particular close to interfaces, due to constraints imposed to the motion of the chains by the rigid nanoparticles; at the same time, dynamics may become faster as a result of increase of free volume due to loosened molecular packing of the chains (plasticization). Fluorescence measurements in selected polymer nanocomposites showed an increase, a decrease or practically no change of $T_{\mathrm{g}}$ for different combinations of matrix and filler and these results were discussed in terms of the type of polymer-filler interaction and of the wetting conditions [11].

$\mathrm{Ag} / \mathrm{PMMA}$ nanocomposites have been synthesized and investigated by several researchers, in particular with respect to their optical and electrical properties, e.g. [1,1214]. A historical survey on metal and semiconductor nanoparticles, polymer nanocomposites based upon them and the importance of small particle sizes on their optical properties has been given by Caseri [15]. Recent progress in synthesis of $\mathrm{Ag}$ nanoparticles and silver/polymer nanocomposites has been reviewed by An et al. [16].
Regarding glass transition and polymer dynamics,Zaragoza-Contreras et al. [17] observed by DSC a notable increase in glass transition temperature $T_{\mathrm{g}}$ in silver/ carbon-PMMA nanocomposites despite low filler fractions (up to $0.1 \mathrm{wt} \%$ ). DMTA measurements on the same samples indicated improvement of mechanical properties, which was related to polymer-filler interfacial interaction [17]. In consistency with that, spectroscopic studies in Ag/poly(2-acrylaimdo-2-methylpropanesulfonic acid-co-methyl methacrylate) nanocomposites indicated specific interaction between the carbonyl groups in the copolymer structure and the $\mathrm{Ag}$ nanoparticles [14]. Polymer-filler interaction, either chemisorption of $\mathrm{CN}$ groups and concomitant formation of a $\sigma$-complex or interaction of carboxylic groups with the Ag nanoparticles, was considered responsible also for an increase of $T_{\mathrm{g}}$ and a decrease of the clearing temperature in nanocomposites of $\mathrm{Ag}$ and a nematic comb-shaped liquid crystalline copolymer with mesogenic cyanobiphenyl side groups and functional units of acrylic acid [18]. On the other hand, DSC measurements in Ag/PMMA nanocomposites showed a decrease of $T_{\mathrm{g}}$, whereas at the same time thermal stability was improved, always with respect to neat PMMA [1]. Please note, however, that, in contrast to the other studies mentioned above, the Ag nanoparticles in Ref. [1] were surface modified with oleylamine.

Extending this brief introduction to include other metal nanoparticles and/or other polymer matrices, it is interesting to mention that systematic studies of the effects of nanoparticles on the properties of the polymer matrix in Pd/PMMA nanocomposites, again at low filler fractions, showed decrease of $T_{\mathrm{g}}$ and of elastic modulus and significant improvement of thermal stability [19]. Increase of the storage modulus was observed in $\mathrm{Ag}$ / poly(vinyl alcohol) nanocomposites [20]. DMTA measurements in $\mathrm{Ag} /$ polyurethane nanocomposites showed that the segmental $\alpha$ relaxation associated with the glass transition becomes slower (i.e. $T_{\mathrm{g}}$ increases) [21]. On the contrary, a depression of $T_{\mathrm{g}}$ was observed in both $\mathrm{Ag} / \mathrm{epoxy}$ and $\mathrm{Al} /$ epoxy nanocomposites, which was related with enhanced polymer dynamics due to extra free volume at the resin-filler interface [22]. Finally, comparative DSC and gas permeability studies in $\mathrm{Ag} /$ polyimide and $\mathrm{Pd} /$ polyimide hybrids indicated weak polymer-metal interaction in the former against strong one in the latter [23].

It becomes clear from the brief survey above that further and more focused work is needed to clarify effects of nanoparticles on glass transition and polymer dynamics in Ag/PMMA nanocomposites. An additional point which should be properly considered is that in metal/polymer nanocomposites typically the matrix is polymerized in the presence of the metal nanoparticles or their precursor $[1,16]$. As a result of that, polymerization conditions for the nanocomposites are different than for the neat polymer, which would affect the final properties [24]. Molar mass determination in the Pd/PMMA nanocomposites mentioned above indicated that MMA polymerization was not affected by the presence of Pd acetate, the precursor for Pd nanoparticle formation [19]. 


\section{Experimental details}

\subsection{Materials}

The synthesis of the Ag/PMMA nanocomposites studied in this work has been described in Ref. [1]. First, Ag nanoparticles were synthesized in water from silver nitrate using sodium borohydride as reducing agent and transferred into chloroform using oleylamine as transfer agent. In the second step, the surface modified Ag nanoparticles were encapsulated in PMMA by in situ radical polymerization of MMA. Samples used for measurements in the present study were in the form of sheets of about $0.7 \mathrm{~mm}$ thickness. The fraction of $\mathrm{Ag}$ nanoparticles in the final materials was varied between 0 and $0.5 \mathrm{wt} \%$. Using typical density values for bulk $\mathrm{Ag}\left(10.5 \mathrm{gr} / \mathrm{cm}^{3}\right)$ and PMMA $\left(1.15 \mathrm{gr} / \mathrm{cm}^{3}\right)$, this corresponds to filler volume fractions between $0 \%$ and $0.055 \%$. In the following the nanocomposites are designated by PMMA/ $X \% \mathrm{Ag}$, where $X$ the fraction of $\mathrm{Ag}$ in wt\%. All the compositions investigated are listed in Table 1.

Number average molar masses (around 250,000 g/mol) and polydispersity indices (around seven), determined by gel permeation chromatography, showed no systematic variation with Ag fraction [1]. TEM measurements on the Ag colloids in chloroform showed that the Ag nanoparticles are spherical with a mean diameter of $5.6 \mathrm{~nm}$. Analysis of UV-vis-spectroscopy measurements indicated the formation of loose Ag aggregates (pairs and squares) in the nanocomposites [1].

\subsection{Apparatus}

Differential scanning calorimetry (DSC) measurements were performed using a DSC 823e apparatus (Mettler Toledo). Nitrogen gas was purged through the DSC cell with a flow rate of $20 \mathrm{ml} / \mathrm{min}$. The weight of the sample varied in the range $6-9 \mathrm{mg}$. In order to erase the effects of any previous thermal history the samples were heated to $180^{\circ} \mathrm{C}$ and then subjected to a cooling scan down to $0^{\circ} \mathrm{C}$ at $20 \mathrm{~K} / \mathrm{min}$, followed by a heating scan up to $180^{\circ} \mathrm{C}$ at a rate of $10 \mathrm{~K} / \mathrm{min}$.

A Seiko DMS 210 dynamic-mechanical analyser was used to perform dynamic mechanical-thermal analysis (DMTA) measurements at $1 \mathrm{~Hz}$. The temperature dependence of storage modulus $\left(E^{\prime}\right)$, loss modulus $\left(E^{\prime \prime}\right)$ and loss tangent $(\tan \delta)$ was measured from 50 to $250{ }^{\circ} \mathrm{C}$ at a heating rate of $2 \mathrm{~K} / \mathrm{min}$. Samples for DMTA measurements were in the form of rectangular sheets of $0.7 \mathrm{~mm}$ thickness, $30 \mathrm{~mm}$ length and $4 \mathrm{~mm}$ width.For dielectric relaxation spectroscopy (DRS) measurements [25] the complex dielectric function (also known as dielectric permittivity or dielectric constant), $\varepsilon^{*}(\omega)=\varepsilon^{\prime}(\omega)-i \varepsilon^{\prime \prime}(\omega)$, was determined as a function of frequency $\left(10^{-2}-10^{7} \mathrm{~Hz}\right)$ at constant temperature ( -150 to $170{ }^{\circ} \mathrm{C}$, controlled to better than $\pm 0.1^{\circ} \mathrm{C}$ ), using a Novocontrol Alpha Analyzer in combination with a Novocontrol Quatro Cryosystem. The samples were circular films of typically $12 \mathrm{~mm}$ diameter and about $0.7 \mathrm{~mm}$ thickness and were placed between brass electrodes.

Thermally stimulated depolarization currents (TSDC) is a dielectric technique in the temperature domain, which consists of measuring the thermally activated release of stored dielectric polarization and is characterized by high sensitivity and high resolving power [26]. In terms of DRS, TSDC corresponds to measuring dielectric loss at a constant low frequency in the range $10^{-4}-10^{-2} \mathrm{~Hz}$. By this technique the sample is inserted between the plates of a capacitor and polarized by the application of an electric field $E_{\mathrm{p}}$ at temperature $T_{\mathrm{p}}$ for time $t_{\mathrm{p}}$, which is large compared to the relaxation time of the dielectric relaxation under investigation. With the electric field still applied, the sample is cooled to a temperature $T_{o}$, which is sufficiently low to prevent depolarization by thermal energy, and then is short-circuited and reheated at a constant rate $b$. The discharge current generated during heating is measured as a function of temperature with a sensitive electrometer. Measurements were carried out using a Keithley 617 electrometer in combination with a Novocontrol sample cell for TSDC measurements. Typical experimental conditions were $115^{\circ} \mathrm{C}$ for $T_{\mathrm{p}}, 7.5 \mathrm{kV} / \mathrm{cm}$ for $E_{\mathrm{p}}, 5 \mathrm{~min}$ for $t_{\mathrm{p}}, 10 \mathrm{~K} /$ min for the cooling rate to $T_{o}=-150^{\circ} \mathrm{C}$, and $3 \mathrm{~K} / \mathrm{min}$ for the heating rate $b$ to $150{ }^{\circ} \mathrm{C}$. Samples and electrodes were similar to those used for DRS measurements.

\section{Results}

Comparative results of DMTA measurements on PMMA and four nanocomposites are shown in Fig. 1: storage modulus $E^{\prime}$ and loss tangent $\tan \delta$ as functions of temperature, focused on the region of the glass transition, at a fixed frequency of $1 \mathrm{~Hz}$. E' decreases slightly in the nanocomposites as compared to the neat PMMA matrix, both in the glassy and in the rubbery state. The glass transition temperature $T_{\mathrm{g}}$, determined as the peak temperature in Fig. 1(b), i.e. at a frequency of $1 \mathrm{~Hz}$ (dynamic glass transition [2]), decreases slightly with increasing filler content from $102^{\circ} \mathrm{C}$ in neat PMMA to $93{ }^{\circ} \mathrm{C}$ in the nanocomposite with the highest amount of filler, $0.5 \mathrm{wt} \%$ (Table 1 ).

DSC measurements were performed in all the compositions studied (Table 1 ) to follow the glass transition. $T_{\mathrm{g}}$ (midpoint) was found to systematically decrease with increasing Ag content (Table 1) and similar trends were

Table 1

Weigth and volume filler fraction of studied nanocomposites together with results for $T_{\mathrm{g}}$ obtained by DMTA, DSC and TSDC.

\begin{tabular}{llllll}
\hline Sample & $\mathrm{Ag}$ content wt $\%$ & $\mathrm{Ag}$ content vol\% & $T_{\mathrm{g}}(\mathrm{DMTA})\left({ }^{\circ} \mathrm{C}\right)$ & $T_{\mathrm{g}}(\mathrm{DSC})\left({ }^{\circ} \mathrm{C}\right)$ & $\Delta T_{\mathrm{g}}(\mathrm{DSC})\left({ }^{\circ} \mathrm{C}\right)$ \\
\hline PMMA & 0 & 0 & 113 & 105 & 18 \\
PMMA/0.0625\%Ag & 0.0625 & 0.0068 & 112 & 104 & 18 \\
PMMA/0.125\%Ag & 0.125 & 0.0137 & 110 & 102 & 17 \\
PMMA/0.25\%Ag & 0.25 & 0.0274 & 108 & 100 & 99 \\
PMMA/0.5\%Ag & 0.5 & 0.055 & 104 & 98 & 16 \\
\hline
\end{tabular}




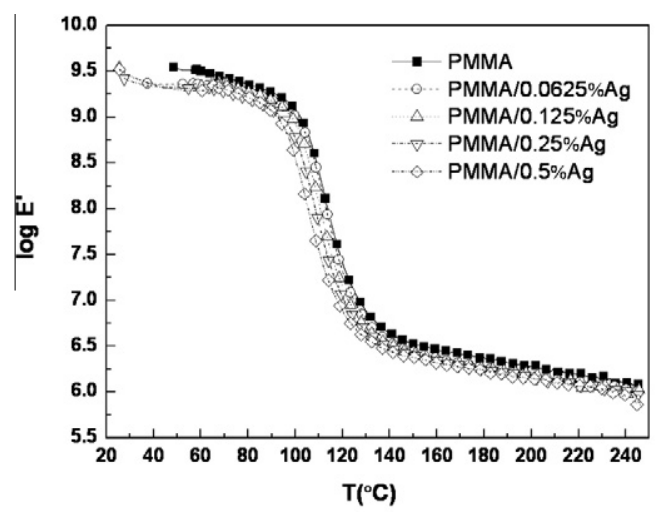

(a)

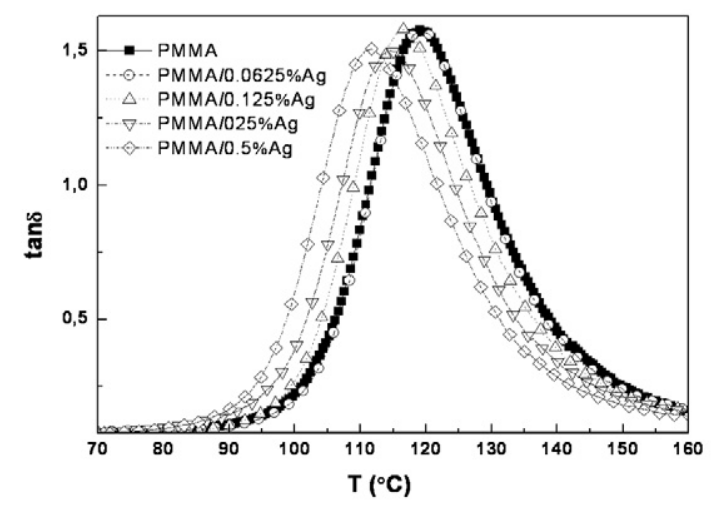

(b)

Fig. 1. Storage modulus $E^{\prime}$ (a) and loss tangent $\tan \delta$ (b) at $1 \mathrm{~Hz}$ against temperature for the samples indicated on the plot.

observed also for the onset and the completion temperature [10]. The width of the glass transition, $\Delta T_{\text {g, }}$ determined as the difference of these last two temperatures [10], does not change, within experimental error $\left( \pm 2{ }^{\circ} \mathrm{C}\right)$, with composition (Table 1 ). Also the heat capacity jump at the glass transition (around $0.28 \mathrm{~J} / \mathrm{g}{ }^{\circ} \mathrm{C}$ ) was found independent of composition.

Molecular dynamics was followed in all the compositions studied by DRS at room temperature and by TSDC. DRS results are shown in Fig. 2: real and imaginary part of the dielectric function, $\varepsilon^{\prime}$ (Fig. 2a) and $\varepsilon^{\prime \prime}$ (Fig. 2b), respectively, as functions of frequency. Two relaxations are observed in the frequency range of measurements, indicated by steps (drops) in $\varepsilon^{\prime}$ and peaks (shoulders) in $\varepsilon^{\prime \prime}$. Comparison with literature $[27,28]$ clearly shows that the strong relaxation centered at around $10 \mathrm{~Hz}$ is the $\beta$ relaxation, associated with the motion of the entire ester moiety $\left(-\mathrm{COOCH}_{3}\right)$, and the weaker shoulder at $10^{5}$ $10^{6} \mathrm{~Hz}$ is the $\gamma$ relaxation, associated with the rotation of the $\alpha$-methyl group bound to the main chain [29]. The main result here is that, within experimental error, no variation of the position (time scale) of neither of the two relaxations in the nanocomposites as compared to the neat PMMA matrix is observed. The magnitude of both relaxations (relaxation strength), on the contrary, increases slightly but systematically with increasing Ag content.

TSDC results for all the compositions studied are shown in Fig. 3 in the form of depolarization current density against temperature, so that results for different samples can be compared to each other, not only with respect to the temperature position of a peak (time scale of the corresponding relaxation), but also with respect to its magnitude (relaxation strength of the corresponding relaxation). In addition to the secondary $\beta$ relaxation, at about $-40{ }^{\circ} \mathrm{C}$, followed by DRS at room temperature (Fig. 2), also the primary (segmental) $\alpha$ relaxation associated with the glass transition and a faster relaxation, $\gamma^{\prime}$, tentatively associated with the presence of traces of water in the sample [30], are followed here, at about 100 and $-130^{\circ} \mathrm{C}$, respectively. In agreement with DRS results in Fig. 2, the peak temperature (time scale) of the secondary $\beta$ relaxation does not change with composition, whereas the magnitude of the peak, which is a measure of the relaxation strength, increases slightly but systematically with

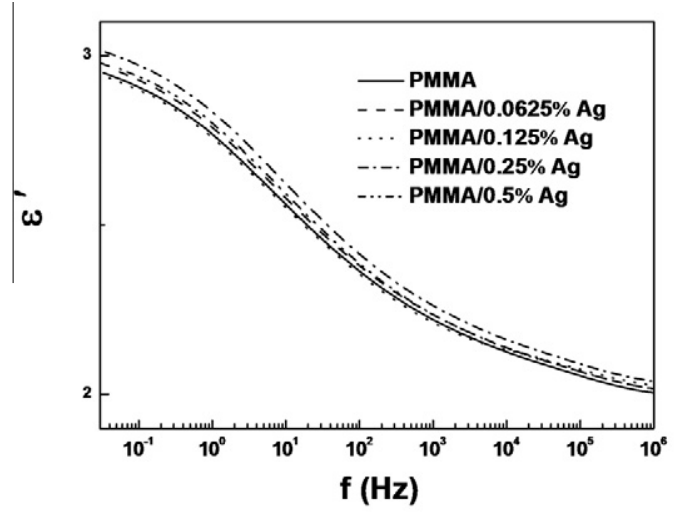

(a)

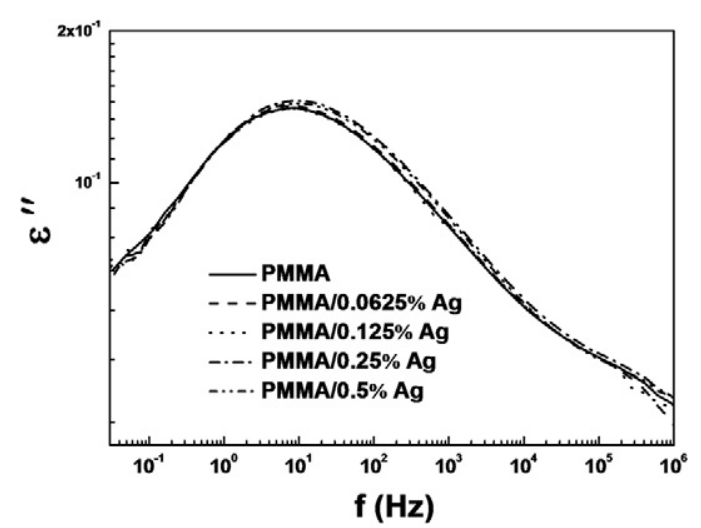

(b)

Fig. 2. Real part of the dielectric function $\varepsilon^{\prime}$ (a) and imaginary part of the dielectric function $\varepsilon^{\prime \prime}$ (b) against frequency $f$ measured at room temperature for the samples indicated on the plot. 


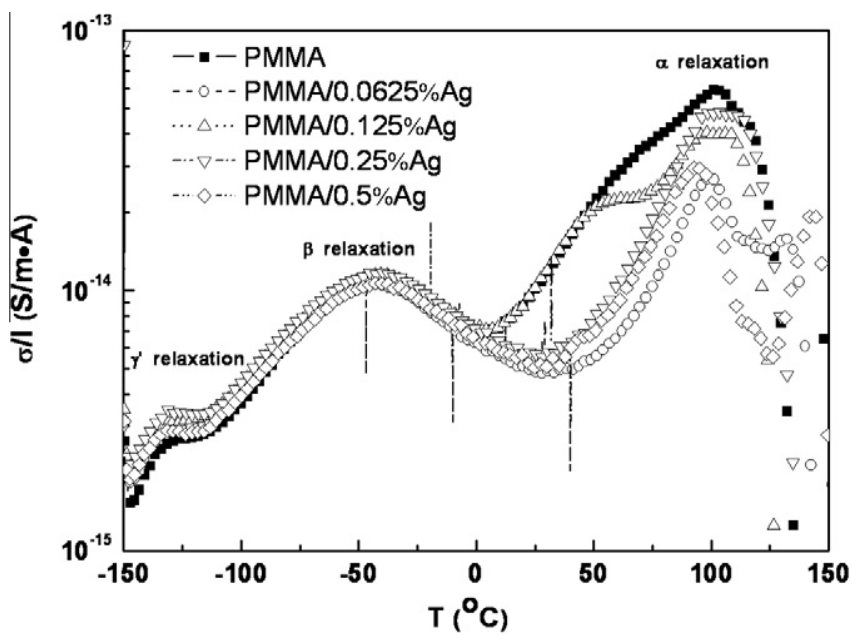

increasing Ag content. The $\gamma$ relaxation, observed by DRS measurements in Fig. 2, is shifted to lower temperatures, out of the temperature range of TSDC measurements in this work [30]. A strong and complex TSDC signal is observed in the temperature region of the glass transition. It is well established from previous work on various acrylates and methacrylates $[30,31]$ that, in addition to the $\alpha$ relaxation, various relaxations associated with charge carrier motion and trapping/detrapping processes contribute to the TSDC response in the temperature region of the glass transition. That is also the reason why the TSDC response is stronger in the region of the $\alpha$ relaxation, as compared to the $\beta$ relaxation, although it is well established $[27,28]$ and it will be confirmed also in this work that $\beta$ is dielectrically stronger than $\alpha$ (and vice versa in DMTA [27]). Nevertheless, it is possible to determine from these measurements (and additional similar measurements with variation of the polarization temperature $T_{\mathrm{p}}$ [26,31], not shown here) the peak temperature of the $\alpha$ relaxation. This temperature, listed in Table 1 , is a good measure of the calorimetric $T_{\mathrm{g}}$ [26], mainly because of the approximately similar time scale of DSC and TSDC, as will be discussed in the next section.

Isothermal DRS measurements in a wide temperature range, from -150 to $170{ }^{\circ} \mathrm{C}$ in steps of 5 or $10^{\circ} \mathrm{C}$, depending on the relaxation under investigation, allowed to follow in detail the evolution of the three relaxations, $\gamma, \beta$ and $\alpha$, as well as of conductivity, in pure PMMA and in the nanocomposite with the highest Ag content, PMMA/0.5\%Ag. Examples are shown in Figs. 4-6 in the form of $\varepsilon^{\prime \prime}(f)$ plots at several temperatures in the temperature region of the $\gamma, \beta$ and $\alpha$ relaxations, respectively, for the neat PMMA matrix. In the inset to each figure comparative $\varepsilon^{\prime \prime}(f)$ plots are shown for the corresponding relaxation in neat PMMA and PMMA/0.5\%Ag at a selected temperature. The $\gamma$ relaxation is presented as a weak and broad relaxation (Fig. 4), the $\beta$ relaxation dominates the dielectric response (Figs. 5 and 6 ) and the $\alpha$ relaxation appears as a weak shoulder between the $\beta$ relaxation at higher and the conductivity wing at lower frequencies (Fig. 6). The comparative plots in the insets demonstrate, in agreement with the results in Figs.
2 and 3, similar time scale and slightly larger magnitude in the nanocomposite for the secondary $\gamma$ and $\beta$ relaxations (Figs. 4 and 5, respectively) and a slight shift of the $\alpha$ relaxation to higher frequencies in the nanocomposite as compared to neat PMMA (Fig. 6).

An overview over the three relaxations in the two samples, neat PMMA and PMMA/0.5\%Ag, is given by the comparative isochronal (constant frequency) $\varepsilon^{\prime \prime}(T)$ plot in Fig. 7. A low frequency, $1.2 \mathrm{~Hz}$, has been selected for that plot for maximizing the peak resolving power and for direct comparison with DMTA $(f=1 \mathrm{~Hz})$ and TSDC (equivalent frequency in the range $10^{-4}-10^{-2} \mathrm{~Hz}$ [26]). The plot confirms the results for the three relaxations described above, showing more clearly than the corresponding isothermal $\varepsilon^{\prime \prime}(f)$ plots, e.g. in the inset to Fig. 6, the acceleration of the $\alpha$ relaxation in the nanocomposite as compared to neat PMMA. Also the magnitude of the $\alpha$ relaxation is larger in the nanocomposite. An additional result in Fig. 7 is that conductivity, which gives rise to the steep increase of $\varepsilon^{\prime \prime}$ at high temperatures [25], is larger in the nanocomposite, in agreement with the comparative $\varepsilon^{\prime \prime}(f)$ plot in the inset to Fig. 6 .

The DRS data were further analyzed by fitting model functions to the experimental data, similar to those shown in Figs. 4-6, to extract quantitative information, in particular for the temperature dependence of the time scale of each relaxation. That was necessary, at least for the $\beta$ and $\alpha$ relaxations (which are the two relaxations of particular interest here), because of overlapping of the two and of the latter with the conductivity wing at lower frequencies (Fig. 6). In that case a sum of two Havriliak-Negami (HN) expressions, one for $\beta$ and one for $\alpha$, and a term of conductivity [32]

$\varepsilon^{\prime \prime}(\omega)=\operatorname{Im}\left(\varepsilon_{\infty}+\sum_{j=1,2}\left(\frac{\Delta \varepsilon_{j}}{\left(1+\left(i \omega \tau_{j}\right)^{a_{j}}\right)^{\beta_{j}}}\right)\right)+K \omega^{-s}$

was fitted to the experimental data. In this equation $\Delta \varepsilon=\varepsilon_{s}-\varepsilon_{\infty}$ is the dielectric strength with $\varepsilon_{s}$ and $\varepsilon_{\infty}$ being the limits of $\varepsilon^{\prime}(\omega)$ for $\omega \tau \ll 1$ and $\omega \tau \gg 1$, respectively. The relaxation time $\tau$ is related to the position of loss maximum by the equation $2 \pi f_{\max }=1 / \tau$. The parameters $\alpha_{j}$ and 


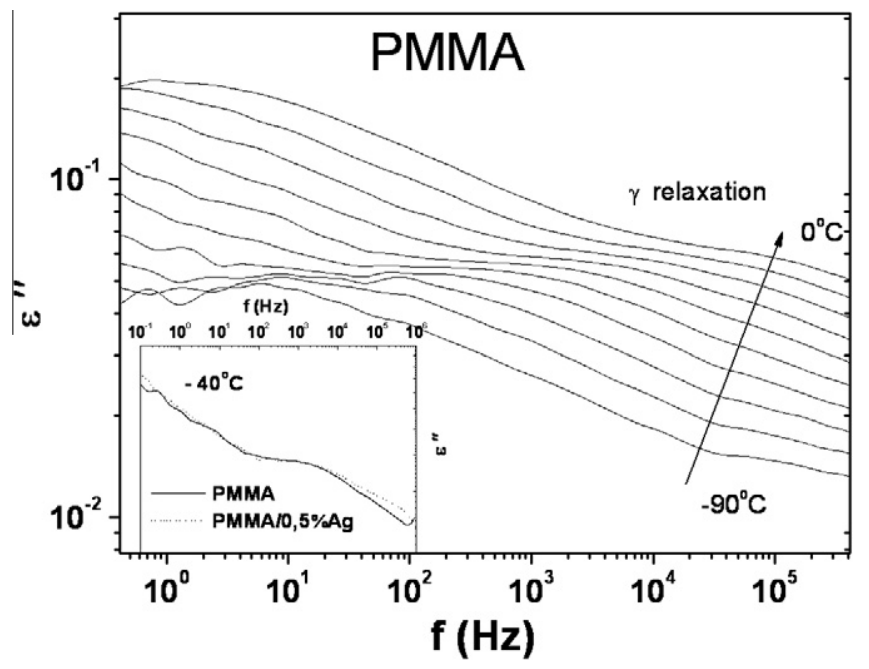

Fig. 4. Isothermal plots of the imaginary part of the dielectric function against frequency, $\varepsilon^{\prime \prime}(f)$, at several temperatures indicated on the plot in the region of the $\gamma$ relaxation in PMMA. The inset shows a comparative $\varepsilon^{\prime \prime}(f)$ plot in neat PMMA and PMMA/0.5\%Ag at $-40{ }^{\circ} \mathrm{C}$.

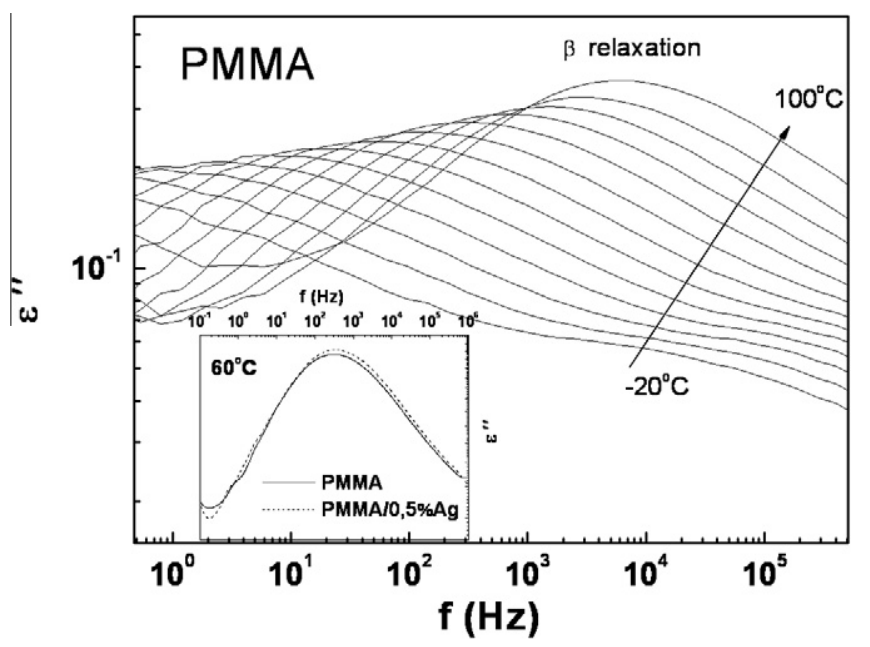

Fig. 5. Isothermal plots of the imaginary part of the dielectric function against frequency, $\varepsilon^{\prime \prime}(f)$, at several temperatures indicated on the plot in the region of the $\beta$ relaxation in PMMA. The inset shows a comparative $\varepsilon^{\prime \prime}(f)$ plot in neat PMMA and PMMA/0.5\%Ag at $60{ }^{\circ} \mathrm{C}$.

$\beta_{j}$ describe the symmetric and asymmetric broadening of the dielectric response, $K$ and $s$ are constants. The analysis provides information on the time scale $(\tau)$, the dielectric strength $(\Delta \varepsilon)$ and the shape $\left(\alpha_{j}, \beta_{j}\right)$ of each relaxation. Here we focus on the time scale of the relaxations.

Time scale is best discussed in terms of the Arrhenius plot (activation diagram) shown in Fig. 8 for PMMA and PMMA/0.5\%Ag. Included in the plot are also DMTA data, at the frequency of measurements of $1 \mathrm{~Hz}$, and TSDC and DSC data, at the equivalent frequencies of $1.6 \times 10^{-3} \mathrm{~Hz}$ (corresponding to a relaxation time of $100 \mathrm{~s}$ [9]) and $2.5 \times 10^{-2} \mathrm{~Hz}$, respectively. The latter was calculated from the approximate expression [33]

$f_{e q}=\frac{b}{2 \pi \alpha \delta T^{\prime}}$

where $b$ is the cooling rate $(10 \mathrm{~K} / \mathrm{min}), \alpha$ is a constant of the order 1 and $\delta T$ is the mean temperature fluctuation (of the order of $2{ }^{\circ} \mathrm{C}$ ). The analysis confirms the observation made above on the basis of the raw data that the time scale of the $\beta$ relaxation does not change in the nanocomposites, whereas the $\alpha$ relaxation becomes faster in the nanoco mposite.

The Arrhenius plot for dc conductivity, $\sigma_{\mathrm{dc}}$, is shown in Fig. 9 for PMMA and PMMA/0.5\%Ag. $\sigma_{\mathrm{dc}}$ was obtained, at each temperature, as the plateau (frequency independent) value of the ac conductivity at low frequencies, calculated from the measured dielectric loss data [25]. The results confirm the observation made already above (Fig. 7) that the conductivity is larger in the nanocomposite (by about a factor of five).

\section{Discussion}

A working hypothesis for explaining the results reported above, to be critically discussed in the following, 


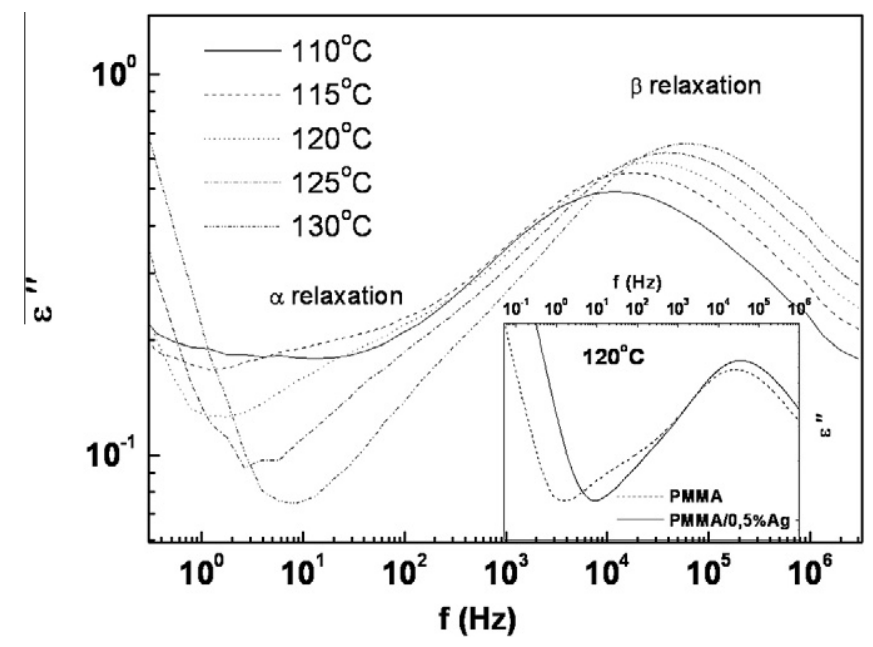

Fig. 6. Isothermal plots of the imaginary part of the dielectric function against frequency, $\varepsilon^{\prime \prime}(f)$, at several temperatures indicated on the plot in the region of the $\beta$ and $\alpha$ relaxations in PMMA. The inset shows a comparative $\varepsilon^{\prime \prime}(f)$ plot in neat PMMA and PMMA $/ 0.5 \% \mathrm{Ag}$ at $120{ }^{\circ} \mathrm{C}$.

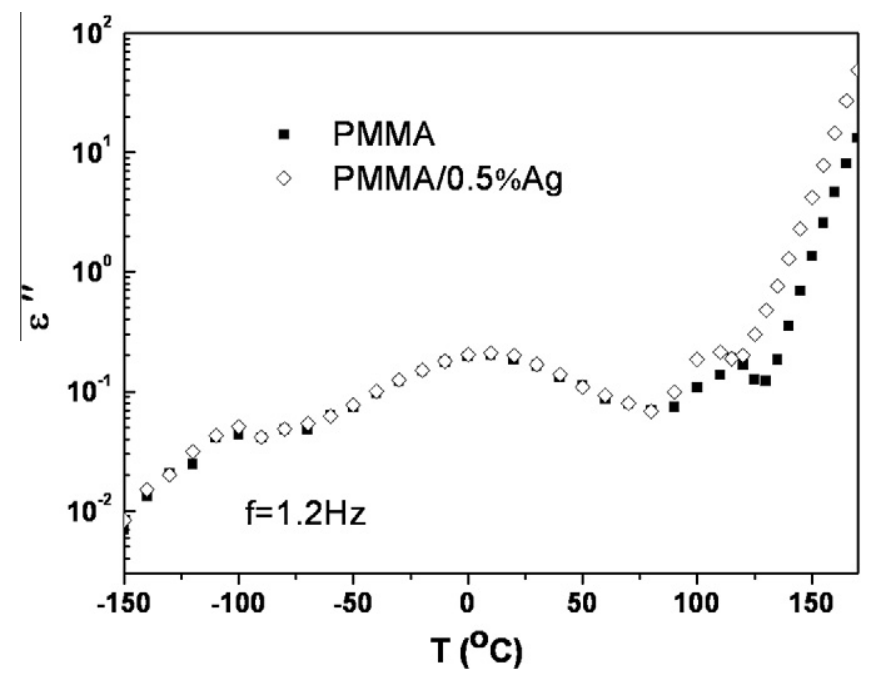

Fig. 7. Isochronal (constant frequency) comparative plots of the imaginary part of the dielectric function against temperature, $\varepsilon^{\prime \prime}(T)$, at $1.2 \mathrm{~Hz}$ in neat PMMA and PMMA/0.5\%Ag.

is that there are no strong polymer-filler interactions in the system under investigation, probably due to the surface modification of the $\mathrm{Ag}$ nanoparticles by oleylamine, whereas the free volume in the nanocomposites increases, due to loosened molecular packing of the polymeric chains near the nanoparticles (plasticization). In the following we will provide further evidence for this hypothesis and we will also quantify results on polymer dynamics and glass transition obtained by the various techniques employed. Correlations between results by various techniques will be also a point of interest.

The secondary $\beta$ relaxation was followed by both DRS and TSDC. Time scale is described, as usually for local, secondary relaxations [25,27], by the Arrhenius equation [2]. Fittings to the experimental data in Fig. 8 are good and, within experimental errors, similar values are obtained for the apparent activation energy $E_{\text {act }}$ in the two samples studied,
PMMA and PMMA/0.5\%Ag, $E_{\text {act }}=0.78$ and $0.80 \mathrm{eV}$, respectively. A change of the slope is observed in Fig. 8, from a lower value at lower temperatures to a slightly higher value at temperatures higher than the calorimetric $T_{\mathrm{g}}$, from the regime $c$ to the regime $b$ in terms of a scenario of merging of the $\alpha$ and $\beta$ relaxations (crossover scenario) characteristic for poly(alkyl methacrylates) [34]. The values of activation energy given above refer to the regime $c$, where the behavior of the $\beta$ relaxation can be characterized without any influence of the cooperative conformational rearrangements of the main chains giving rise to the $\alpha$ relaxation. It is interesting to note with respect to the merging scenario mentioned above that the same change of slope is observed in neat PMMA and the nanocomposite PMMA/0.5\%Ag. Measurements at higher frequencies/temperatures would allow to follow the relaxational behavior in the a regime, where the $\alpha$ and $\beta$ relaxations in PMMA merge in the $\alpha \beta$ process with 


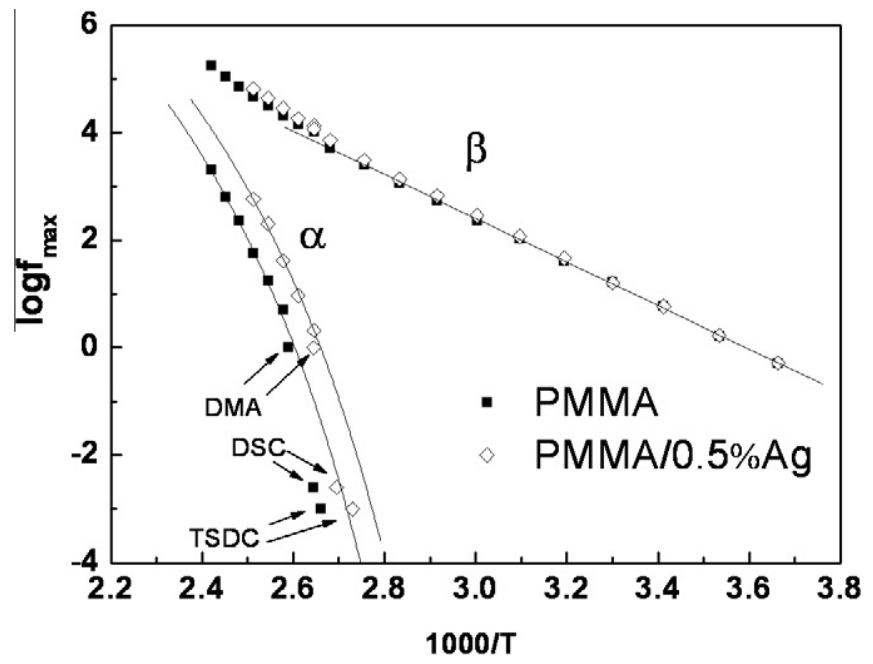

Fig. 8. Arrhenius plot for PMMA and PMMA/0.5\%Ag. Included in the plot are also DMTA, TSDC and DSC data. The lines are fittings of the VTF Eq. (3) to the data for the $\alpha$ relaxation, for both samples, and of the Arrhenius equation to the data for the $\beta$ relaxation and the $\gamma$ relaxation (inset), only for neat PMMA for the seek of clarity.

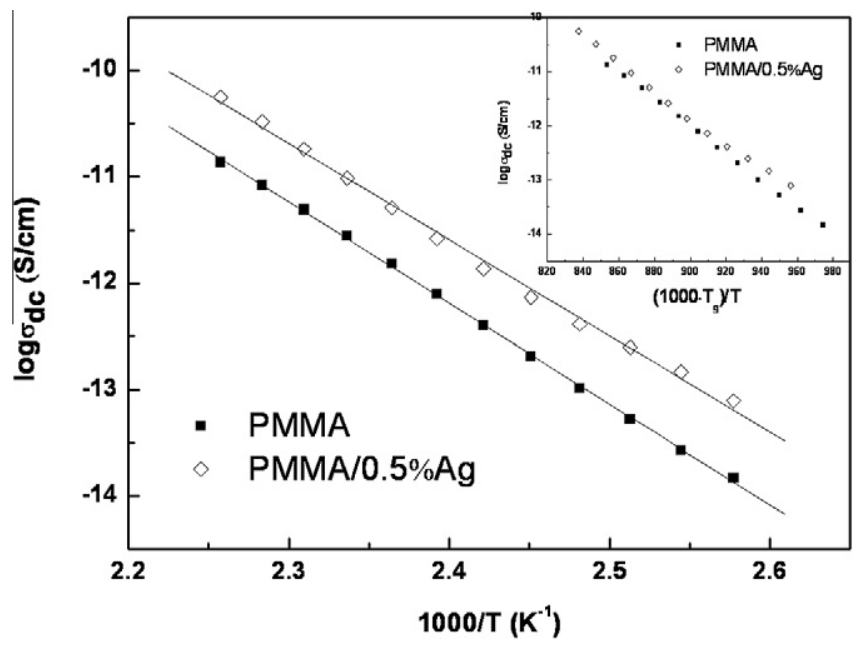

Fig. 9. Arrhenius plot for the dc conductivity $\sigma_{\mathrm{dc}}$ in PMMA and PMMA/0.5\%Ag. The lines are fittings of the Arrhenius equation to the experimental data. The inset shows the $T_{\mathrm{g}}$-scaled corresponding plot.

a higher slope (activation energy) than in the $b$ regime $[28,34]$, and so to study effects of nanoparticles on the overall merging scenario of the $\alpha$ and $\beta$ relaxations in PMMA. Combined DMTA, TSDC and DRS measurements in copolymer networks and interpenetrating polymer networks of PMMA and poly(butyl acrylate) showed changes in the crossover scenario depending on composition and nanostructure [28].

Similar to the secondary $\beta$ relaxation, the primary $\alpha$ relaxation (dynamic glass transition) was followed by the dielectric DRS and TSDC techniques, and in addition by DMTA (Fig. 1). The three techniques show, in agreement to each other, that the relaxation becomes slightly but systematically faster with increasing filler content in the nanocomposites. Effects of nanoparticles on the magnitude of the relaxation are less easy to study, due to overlapping with the $\beta$ relaxation and, mainly, conductivity. The isochronal plots of Fig. 7 show clearly, however, that the relaxation becomes stronger in the nanocomposite PMMA/0.5\%Ag as compared to neat PMMA. In the DMTA measurements the $\alpha$ relaxation is, of course, not affected by electrical conductivity, and, in addition, overlapping with the $\beta$ relaxation is less significant, as, in contrast to dielectric, in mechanical measurements the $\alpha$ relaxation is stronger than the $\beta$ relaxation. The DMTA plots in Fig. 1 show no broadening of the $\tan \delta$ loss peaks in the nanocomposites, which would suggest a broadening of the distribution of relaxation times and a heterogeneous behavior [28].

The results for the time scale of the $\alpha$ relaxation in neat PMMA and the nanocomposite PMMA/0.5\%Ag are summarized in Fig. 8. The Vogel-Tammann-Fulcher (VTF) equation [2] 
$f_{\max }=f_{0} \cdot \exp \left(-\frac{B}{T-T_{o}}\right)$

appropriate for cooperative relaxation processes, like the glass transition, was fitted to the DRS data for the two samples. In this equation $f_{o}, B$ and $T_{o}$ (Vogel temperature) are temperature independent empirical constants. In order to reduce the number of free parameters, $f_{o}$ was fixed to the phonon frequency $10^{13} \mathrm{~Hz}$ [25]. The fittings are satisfactory with reasonable values of the fitting parameters, $B=2674 \mathrm{~K}$ and $T_{o}=294 \mathrm{~K}$ for neat PMMA and $B=2695 \mathrm{~K}$ and $T_{o}=285 \mathrm{~K}$ for PMMA $/ 0.5 \% \mathrm{Ag}$.

It is interesting, from the methodological and the metrological point of view also, to compare with each other results for the $\alpha$ relaxation and the glass transition obtained by various techniques [35]. This can be done here first on the basis of the Arrhenius plot of Fig. 8, where we observe that the $\alpha$ relaxation is slightly faster (shifted to higher temperatures) by DSC, TSDC and DMTA, as compared to DRS. The opposite result was obtained in polymer blends based on polyurethanes, with the $\alpha$ relaxation being slightly faster, up to one decade in frequency, by DRS as compared to DSC/TSDC/DMTA [36]. Comparison with more systems referred to in [28], indicates that the various techniques give different absolute values, depending on the specific system, but identical temperature dependences [37]. Please note that for DMTA both frequency and temperature in Fig. 8 have been obtained directly from the measurements; the loss peak frequency for DRS has been obtained by the fitting analysis described in Section 3, Eq. (1), at the temperature of measurement; finally, for DSC and TSDC the frequencies in Fig. 8 are equivalent frequencies, following from rather rough estimations and calculations, at the temperature of measurement. It should be kept in mind also, that, in order to facilitate comparison with the DRS data, the VTF plots, obtained solely on the basis of the DRS data, had to be extrapolated to temperatures/ frequencies where no DRS data are available.

There is no doubt, however, that, despite slight discrepancies, the data for the $\alpha$ relaxation in Fig. 8 refer all to the same process. To further follow and quantify this point, Fig. 10 presents data for the composition dependence of the glass transition temperature $T_{\mathrm{g}}$, directly determined by DSC, DMTA and TSDC. They show all the same trend of decreasing with increasing filler content (by approximately the same amount, Table 1). The relative shift of the data for the same composition to higher temperatures in the order TSDC, DSC, DMA is in (qualitative) agreement with the shift of the frequency of measurement/presentation in the same order, $1.6 \times 10^{-3}, 2.5 \times 10^{-2}$ and $1 \mathrm{~Hz}$. We can include DRS in this comparison, by calculating $T_{\mathrm{g}}$ diel from the VTF plots in Fig. 8, defined by convention as the temperature where the relaxation time becomes $100 \mathrm{~s} \mathrm{[8]}$. The values obtained are $T_{\mathrm{g} \text { diel }}=96{ }^{\circ} \mathrm{C}$ and $88^{\circ} \mathrm{C}$ for neat PMMA and the nanocomposite PMMA/0.5\% Ag, respectively. In agreement with the data by the other three techniques in Fig. 10, $T_{\mathrm{g} \text { diel }}$ is reduced in the nanocomposite as compared to neat PMMA, by approximately the same amount $\left(8^{\circ} \mathrm{C}\right)$. Absolute values are slightly lower than by TSDC, despite similar time scale, probably because of contribution of conductivity to the TSDC $\alpha$ peak, as discussed above [26,31].

In Fig. 11 we compare directly to each other DMTA and DRS data for PMMA and the nanocomposite PMMA/ 0.5\% Ag: $E^{\prime \prime}(T)$ data measured at $1 \mathrm{~Hz}$ and $\varepsilon^{\prime \prime}(T)$ replotted from isothermal DRS measurements at practically the same frequency, $1.2 \mathrm{~Hz}$. We observe for both compositions higher peak temperatures for the DRS data, by $5-10^{\circ} \mathrm{C}$, i.e. the DRS response is slower than the DMTA response. Please note, however, that, by definition, $\varepsilon^{\prime \prime}$ is a compliance, whereas $E^{\prime \prime}$ a modulus, and that for the same quantity (experimental technique) modulus is faster than compliance [38]. For that reason we transformed the $\varepsilon^{\prime \prime}(T)$ data to electric modulus $M^{\prime \prime}(T)$ data [38] and compared with the $E^{\prime \prime}(T)$ data: the DRS response remains slower than the DMTA response (results not shown here). Interestingly, the relative position of the loss peaks changes in the $\tan \delta(T)$ presentation, the DMTA peaks being now at higher temperatures, obviously because of the different temperature dependence of $E^{\prime}$ and $\varepsilon^{\prime}$ (the former decreasing and the latter increasing with temperature [27]).

The Arrhenius plot of dc conductivity in Fig. 9 shows that $\sigma_{\mathrm{dc}}$ is at each temperature by a factor of about five larger in the nanocomposite as compared to neat PMMA. The temperature dependence of conductivity is described by the Arrhenius equation. The values of the apparent activation energy, $1.8 \mathrm{eV}$ in PMMA and $1.9 \mathrm{eV}$ in PMMA/0.5\% Ag, are within the experimental error $( \pm 0.1 \mathrm{eV})$, identical for the two samples. We should rather expect $\sigma_{d c}(T)$ to be described by a VTF-type equation, as conductivity in polymers above $T_{\mathrm{g}}$ is typically governed by the motion of the polymeric chains $[25,27]$. Such a behavior was in fact observed in nanocomposites of $\mathrm{Ag}$ and a nematic combshaped liquid crystalline copolymer with mesogenic cyanobiphenyl side groups and functional units of acrylic acid [18]. Interestingly, also in that system conductivity was found to increase in the nanocomposites, although $T_{\mathrm{g}}$ also increased, indicating a reduction of segmental dynamics. Despite the Arrhenius dependence of $\sigma_{d c}(T)$ in the materials under investigation, we show in the inset to Fig. 9 the $T_{\mathrm{g}}$-scaled Arrhenius plot (using the $T_{\mathrm{g}}$ values obtained by DSC) to further follow the origin of the increase of $\sigma_{\mathrm{dc}}$ in the nanocomposite. The results show that the data for the two samples come closer together, suggesting that the increase of conductivity in the nanocomposite, at each temperature, arises mostly from the reduction of $T_{\mathrm{g}}$ and the resulting increase of mobility in the nanocomposite at the common temperature of measurements, rather than the increase of charge carrier concentration.

Coming back to the working hypothesis at the beginning of this section, namely that free volume in the nanocomposites increases, due to loosened molecular packing of the polymeric chains near the nanoparticles, a comment is in order. One might argue that another explanation for the results reported above would be that of residual solvent as a plasticizer, such as oleylamine used for surface modification of $\mathrm{Ag}$ nanoparticles. This is true if the solvent is not bound to the polymer (for bound solvent antiplasticization of the secondary relaxations is expected [39], as observed for example in poly(hydroxyethyl acrylate)water mixtures [38]), or if it is in excess. This is, however, 


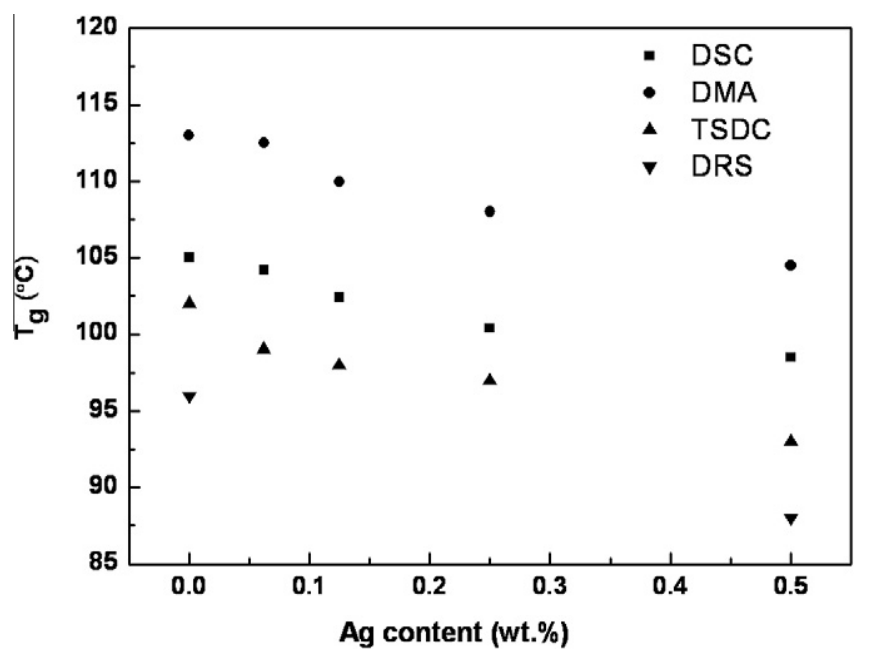

Fig. 10. Glass transition temperature $T_{\mathrm{g}}$, determined by DSC, DMTA, TSDC and DRS against Ag content.

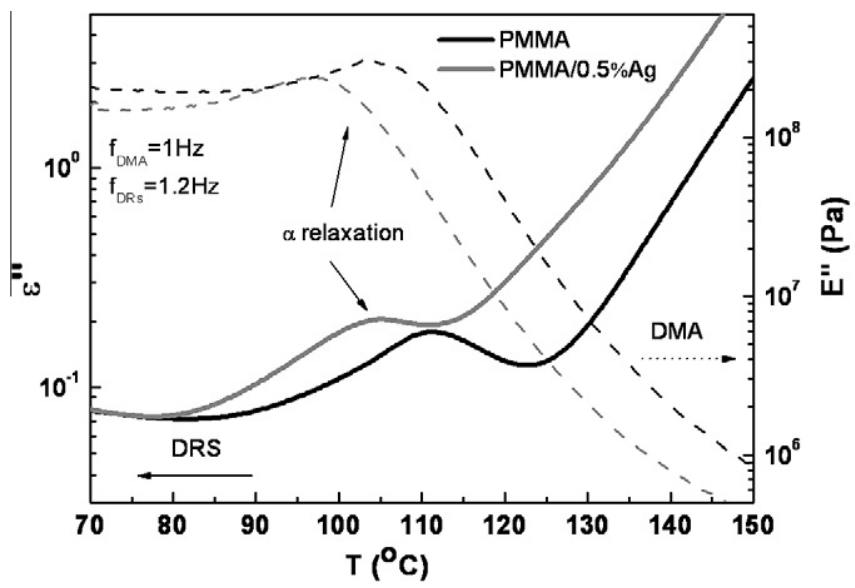

Fig. 11. Isochronal comparative dielectric $\varepsilon^{\prime \prime}(T)$ and mechanical $E^{\prime \prime}(T)$ plots at 1.2 and $1.0 \mathrm{~Hz}$, respectively, in neat PMMA and PMMA/0.5\%Ag.

rather unlikely, because of the high final temperature of measurements by the various techniques $\left(250^{\circ} \mathrm{C}\right.$ by DMTA, $180^{\circ} \mathrm{C}$ by DSC, $150^{\circ} \mathrm{C}$ by TSDC, and $170^{\circ} \mathrm{C}$ by DRS, combined with the presence of dry gas, compare Section 2) and the reproducibility of results obtained in a second heating scan. Please note that this point (plasticization by bulky nanoparticles against plasticization by remaining solvent) was discussed also in Ref. [39].

The final point of discussion refers to the mechanical properties of the nanocomposites. As shown in Fig. 1, the elastic modulus $E^{\prime}$ decreases slightly but systematically in the nanocomposites as compared to the neat PMMA matrix, both in the glassy and in the rubbery state. In addition, practically no change of the area under the $\tan \delta$ loss peaks is observed in the nanocomposites. These results correlate well with the slight decrease of $T_{\mathrm{g}}$ and the acceleration of segmental dynamics in the nanocomposites and the explanation for that in terms of the absence of strong polymerfiller interactions. Similar results, i.e. decrease of both $T_{\mathrm{g}}$ and elastic modulus, have been obtained in Pd/PMMA nanocomposites, at low filler fractions comparable to those of the present study [19]. In consistency with this scheme, both a notable increase in $T_{\mathrm{g}}$ and an improvement of mechanical properties was observed in silver/carbonPMMA nanocomposites, again at low filler fractions (up to $0.1 \mathrm{wt} \%$ ), and these results were related to polymer-filler interfacial interaction [17]. It is interesting to note that thermal stability apparently does not fit into this scheme, as improvement of thermal stability and simultaneous decrease of $T_{\mathrm{g}}$ have been reported in Ag/PMMA [1] and Pd/ PMMA nanocomposites [19].

\section{Conclusions}

Polymer dynamics and glass transition in Ag/PMMA nanocomposites were investigated by the combined use of four complementary techniques, DMTA, DSC, TSDC and DRS. The two main conclusions drawn from the results refer to the correlation between polymer dynamics and 
mechanical properties in polymer nanocomposites and to the origin of the effects of nanoparticles on polymer dynamics at the molecular level.

The results by the four techniques show, in good agreement to each other, an acceleration of segmental techniques and a lowering of $T_{\mathrm{g}}$ in the nanocomposites. At the same time, the elastic modulus decreases slightly but systematically in the nanocomposites, both in the glassy and in the rubbery state. These results indicate a correlation between polymer dynamics and mechanical properties, supported also by results in the literature reported above. It would be interesting in future work to make use of the detailed picture of polymer dynamics in nanocomposites we may get from dielectric techniques for modeling and predicting the mechanical properties of interest.

The second main conclusion refers to the origin of the effects of nanoparticles on polymer dynamics at the molecular level. A general scheme of thinking about the results reported in this work and similar results in the literature is in terms of two main contradictory effects of nanoparticles on chain dynamics, namely a slowing down of dynamics, in particular close to interfaces, resulting from specific polymer-filler interactions, on the one hand, and an acceleration of dynamics, on the other hand, as a result of increase of free volume due to loosened molecular packing of the chains (plasticization). The second effect of plasticization should be a general one in polymer nanocomposites, so that the overall behavior is determined by the presence and strength of specific polymer-filler interactions. Thus, the overall increase of molecular mobility in the nanocomposites under investigation should result from the absence of strong polymer-filler interactions, obviously due to the surface modification of the Ag nanoparticles by oleylamine. Work in progress on other polymer nanocomposites with the same matrix (PMMA) and different fillers, carbon nanotubes (CNT) and silica nanoparticles, provide further evidence for this scheme of explanation. In the PMMA/CNT nanocomposites, prepared by melt-mixing using non-functionalized CNT, the secondary relaxations, the $\alpha$ relaxation, $T_{\mathrm{g}}$, and the elastic modulus remained all unaffected by CNT ( $0-8$ wt\%). These results would suggest, in addition to the absence of strong polymer-filler interactions, negligible effects of plasticization, probably due to the shape and flexibility of CNT. In PMMA/silica nanocomposites, on the other hand, with well-established strong interactions (hydrogen bonding between the polymer carbonyls and the silica hydroxyls), the $\alpha$ relaxation becomes slower and both $T_{\mathrm{g}}$ and the elastic modulus increase significantly.

\section{Acknowledgements}

The research leading to these results has received partial support from the European Community's Seventh Framework Programme (FP7/2007-2013) under Grant Agreement No. 218331 NaPolyNet.

JLGR acknowledges the support of the Spanish Ministry of Science and Innovation through project No. EUI200800126 and funding in the Centro de Investigación Principe Felipe in the field of Regenerative Medicine through the collaboration agreement from the Conselleria de Sanidad (Generalitat Valenciana), and the Instituto de Salud Carlos III (Ministry of Science and Innovation).

\section{References}

[1] Vodnik VV, Božanić DK, Džunuzović E, Vuković J, Nedeljković JM. Thermal and optical properties of silver-poly(methyl methacrylate) nanocomposites prepared by in-situ radical polymerization. Eur Polym J 2010;46(2):137-44.

[2] Donth E. The glass transition: relaxation dynamics in liquids and disordered materials. Berlin: Springer; 2001.

[3] Scheidler P, Kob W, Binder K. The relaxation dynamics of a supercooled liquid confined by rough walls. J Phys Chem B 2004;108(21):6673-86.

[4] Coleman JN, Cadek M, Ryan KP, Fonseca A, Nagy JB, Blau WJ, et al. Reinforcement of polymers with carbon nanotubes. The role of an ordered polymer interfacial region. Experiment and modeling. Polymer 2006;47(26):8556-61.

[5] Papakonstantopoulos GJ, Yoshimoto K, Doxastakis M, Nealey PF, De Pablo JJ. Local mechanical properties of polymeric nanocomposites. Phys Rev E 2005;72(3):1-6.

[6] He F, Wang LM, Richert R. Dynamics of supercooled liquids in the vicinity of soft and hard interfaces. Phys Rev B 2005;71(14):1-10.

[7] Alcoutlabi M, McKenna GB. Effects of confinement on material behaviour at the nanometre size scale. J Phys Condens Matter $2005 ; 17(15)$.

[8] Fragiadakis D, Pissis P, Bokobza L. Glass transition and molecular dynamics in poly(dimethylsiloxane)/silica nanocomposites. Polymer 2005;46(16):6001-8.

[9] Fragiadakis D, Pissis P. Glass transition and segmental dynamics in poly(dimethylsiloxane)/silica nanocomposites studied by various techniques. J Non-Cryst Solids 2007;353(47-51):4344-52.

[10] Bershtein VA, Egorova LM, Yakushev PN, Pissis P, Sysel P, Brozova L. Molecular dynamics in nanostructured polyimide-silica hybrid materials and their thermal stability. J Polym Sci Part B Polym Phys 2002;40:1056-69.

[11] Rittigstein P, Torkelson JM. Polymer-nanoparticle interfacial interactions in polymer nanocomposites: confinement effects on glass transition temperature and suppression of physical aging. J Polym Sci Part B Polym Phys 2006;44(20):2935-43.

[12] Basak D, Karan S, Mallik B. Significant modifications in the electrical properties of poly(methyl methacrylate) thin films upon dispersion of silver nanoparticles. Solid State Commun 2007;141(9):483-7.

[13] Singh N, Khanna PK. In situ synthesis of silver nano-particles in polymethylmethacrylate. Mater Chem Phys 2007;104(2-3):367-72.

[14] Li D, Xu G, Tao Y. Studies on the interaction between nano-Ag and P(AMPS-MMA). Acta Polym Sin 2008(4):378-82.

[15] Caseri W. Nanocomposites of polymers and metals or semiconductors: historical background and optical properties. Macromol Rapid Commun 2000;21(11):705-22.

[16] An J, Wang D, Luo Q, Li X, Li M, Yuan X. Silver/polymer nanocomposites. Prog Chem 2008;20(6):859-68.

[17] Zaragoza-Contreras EA, Hernández-Escobar CA, Mendoza-Duarte ME, Flores-Gallardo SG, Barra-Gómez R, Márquez-Lucero A. Thermal and mechanical analysis of silver/carbon nanoparticlePMMA nanocomposites obtained by miniemulsion polymerization. Polym J 2009;41(10):816-21.

[18] Nikonorova NA Barmatov EB, Pebalk DA, Barmatova MV, Domínguez-Espinosa G, Diaz-Calleja R, et al. Electrical properties of nanocomposites based on comb-shaped nematic polymer and silver nanoparticles. J Phys Chem C 2007;111(24):8451-8.

[19] Aymonier C, Bortzmeyer D, Thomann R, Mülhaupt R. Poly(methyl methacrylate)/palladium nanocomposites: synthesis and characterization of the morphological, thermomechanical, and thermal properties. Chem Mater 2003;15(25):4874-8.

[20] Gautam A, Ram S. Preparation and thermomechanical properties of Ag-PVA nanocomposite films. Mater Chem Phys 2010;119(12):266-71.

[21] Cocca M, D’Orazio L. Novel silver/polyurethane nanocomposite by in situ reduction: effects of the silver nanoparticles on phase and viscoelastic behavior. J Polym Sci Part B Polym Phys 2008;46(4):344-50.

[22] Sun Y, Zhang Z, Moon KS, Wong CP. Glass transition and relaxation behavior of epoxy nanocomposites. J Polym Sci Part B Polym Phys 2004;42(21):3849-58.

[23] Compton J, Thompson D, Kranbuehl D, Ohl S, Gain O, David L, et al. Hybrid films of polyimide containing in situ generated silver or 
palladium nanoparticles: effect of the particle precursor and of the processing conditions on the morphology and the gas permeability. Polymer 2006; 47(15):5303-13.

[24] Maroulas P, Kripotou S, Pissis P, Fainleib A, Bei I, Bershtein V, et al. Molecular mobility in polycyanurate/clay nanocomposites studied by dielectric techniques. J Compos Mater 2009;43(9):943-58.

[25] Kremer F, Schönhals A. Broadband dielectric spectroscopy. Berlin: Springer; 2002.

[26] Van Turnhout J. Thermally stimulated discharge of electrets. In: Sessler GM, editor. Topics in applied physics, electrets. Berlin: Springer; 1980. p. 81-215.

[27] McCrun NG, Read BE, Williams G. Anelastic and dielectric effects in polymeric solids. Berlin: Springer; 1991.

[28] Espadero Berzosa A, Gómez Ribelles JL, Kripotou S, Pissis P. Relaxation spectrum of polymer networks formed from butyl acrylate and methyl methacrylate monomeric units. Macromolecules 2004;37(17):6472-9.

[29] Schmidt-Rohr K, Kulik AS, Beckham HW, Ohlemacher A, Pawelzik U, Boeffel C, et al. Molecular nature of the $\beta$ relaxation in poly(methyl methacrylate) investigated by multidimensional NMR. Macromolecules 1994;27(17):4733-45.

[30] Kalogeras IM. Thermally stimulated currents of poly(methyl methacrylate): comments on the molecular origin of a Debye-type signal between the $\alpha$ - and $\beta$-relaxation modes. J Polym Sci Part B Polym Phys 2004;42(4):702-13.

[31] Kyritsis A, Pissis P, Gómez Ribelles JL, Monleón Pradas MM. Depolarization thermocurrent studies in poly(hydroxyethyl acrylate)/water hydrogels. J Polym Sci Part B Polym Phys 1994;32(6):1001-8.
[32] Havriliak S, Havriliak SJ. Dielectric and mechanical relaxation in materials: analysis, interpretation and application to polymers. Cincinnati: Hanser; 1997.

[33] Hensel A, Dobbertin J, Schawe JEK, Boller A, Schick C. Temperature modulated calorimetry and dielectric spectroscopy in the glass transition region of polymers. J Therm Anal Calorim 1996;46(3):935-54.

[34] Garwe F, Schönhals A, Lockwenz H, Beiner M, Schröter K, Donth E. Influence of cooperative $\alpha$ dynamics on local $\beta$ relaxation during the development of the dynamic glass transition in poly $(n$-alkyl methacrylate)s. Macromolecules 1996;29(1):247-53.

[35] Beiner M, Korus J, Lockwenz H, Schroter K, Donth E. Heat capacity spectroscopy compared to other linear response methods at the dynamic glass transition in poly(vinyl acetate). Macromolecules 1996;29(15):5183-9.

[36] Vatalis AS, Kanapitsas A, Delides CG, Pissis P. Relaxation phenomena and morphology in polymer blends based on polyurethanes investigated by various thermal analysis techniques. Thermochim Acta 2001;372(1-2):33-8.

[37] Santangelo PG, Roland CM. Temperature dependence of mechanical and dielectric relaxation in cis-1,4-polyisoprene. Macromolecules 1998;31(11):3715-9.

[38] Kyritsis A, Pissis P, Grammatikakis J. Dielectric relaxation spectroscopy in poly(hydroxyethyl acrylates)/water hydrogels. J Polym Sci Part B Polym Phys 1995;33(12):1737-50.

[39] Hao N, Böhning M, Schönhals A. Dielectric properties of nanocomposites based on polystyrene and polyhedral oligomeric phenethyl-silsequioxanes. Macromolecules 2007;40:9672-9. 